\title{
A SURVEY OF MASS LOSS FROM Be AND SHELL STARS USING ULTRAVIOLET DATA FROM COPERNICUS
}

\author{
J. M. MARLBOROUGH \\ University of Western Ontario, London, Ontario, Canada \\ and \\ THEODORE P. SNOW, JR. \\ Princeton University Observatory, Princeton, N.J., U.S.A.
}

\begin{abstract}
Ultraviolet spectra of intermediate resolution have been obtained with Copernicus of twelve objects classified as Be or shell stars, and an additional 19 dwarfs of spectral classes B0-B4. Some of these spectra show marked asymmetries in certain resonance lines, especially the Si IV doublet at $\lambda 1400 \AA$, indicating the presence of outflowing material with maximum velocities of nearly $1000 \mathrm{~km} \mathrm{~s}^{-1}$. Direct evidence for mass loss at these velocities is seen for the first time in dwarf stars as late as B1.5. Later than B0.5, the only survey objects showing this phenomenon are Be stars. Among the stars considered there is a correlation between the presence of mass-loss effects and projected rotational velocity, suggesting that the UV flux from B1-B3 dwarfs is sufficient to drive high-velocity stellar winds only if rotation reduces the effective gravity near the equator. The role of mass-loss in producing the Be star phenomenon and the effects of rotation on mass loss are discussed.
\end{abstract}

\section{Introduction}

It is generally accepted that the shell and/or emission lines observed in the spectra of $\mathrm{Be}$ and shell stars arise from a low density extended atmosphere or circumstellar envelope which exhibits both differential rotation and strong concentration to the equatorial plane of the star. From the spectroscopic observations one infers that this circumstellar matter is in motion, most of the motion being rotational, with typical velocities of a few hundred $\mathrm{km} \mathrm{s}^{-1}$ at a distance of several stellar radii above the star's surface. At such distances the velocity of escape from the star is of the same order of magnitude as the observed velocities. Consequently it seems probable that some or all of the observed gas ultimately escapes from the star. General discussions of these and other properties of Be and shell stars are given by McLaughlin (1961) and Underhill (1966), and reviewed by Hack and Struve (1970).

Nevertheless, little direct evidence in support of this widely accepted picture exists. In the optical region the radial component of velocity of the shell material, obtained from the central reversals of hydrogen lines and/or shell lines, is small, generally less than $10-20 \mathrm{~km} \mathrm{~s}^{-1}$. Only during the active phases of some $\mathrm{Be}$ and shell stars does this radial component increase and then it normally does not exceed $100 \mathrm{~km} \mathrm{~s}^{-1}$. Perhaps radial components of velocity of this order of magnitude indicate mass loss during the active phases, although the interpretation of what these velocities mean during these phases is not clear. Ultraviolet data obtained by Heap (1975) using a rocket-borne spectrograph show displaced resonance lines in the spectrum of $\zeta$ Tau corresponding to an outflow velocity of approximately $120 \mathrm{~km} \mathrm{~s}^{-1}$, but whether or not this truly represents mass loss depends on the location of the absorbing material. Similar displacements have been seen by Marlborough (unpublished) in Copernicus spectra of $\alpha$ Eri, 59 Cyg, and HD 28497. 
In the present study we are more concerned with evidence for mass outflow at velocities of several hundred $\mathrm{km} \mathrm{s}^{-1}$, which requires substantial acceleration of the material beyond the photosphere, and which almost certainly represents true mass loss. Bohlin (1970) noted that the $C_{\text {IV }}$ resonance doublet in $\gamma$ Cas has a P Cygni profile with the absorption component displaced by at least $450 \mathrm{~km} \mathrm{~s}^{-1}$ from the stellar rest frame, but additional evidence for high-velocity mass loss from $\mathrm{Be}$ and shell stars has not been forthcoming. It is the purpose of this paper to report the results of a survey of such effects, based on Copernicus spectra of Be and shell stars. A partial description of the Copernicus results has been published elsewhere (Snow and Marlborough 1976).

\section{The Data}

All of the spectra used in this study were acquired with photomultiplier tube U2 (Rogerson et al., 1973) at a nominal resolution of $0.2 \AA$. Backgrounds due to charged particles were subtracted using standard procedures developed at Princeton (Snow et al., 1975) and the stray light contributions were removed following the algorithm of Bohlin (1975). The overall photometric accuracy, with errors dominated by drifts in the spacecraft guidance, is thought to be roughly $10 \%$ of the continuum level. Wavelengths are accurate to about half the U2 bandpass, i.e. $\pm \sim 0.1 \AA$.

For comparison with the $\mathrm{Be}$ and shell stars, all objects of luminosity class IV or V in the spectral class range $\mathrm{B} 0$ to $\mathrm{B} 4$ for which a complete $\mathrm{U} 2$ spectrum is available have been considered. This includes several stars from the mass-loss survey of Snow and Morton (1976). The distribution of these normal stars as well as the $\mathrm{Be}$ and shell stars is depicted in Figure 1, where it is seen that each subclass from B0 to B4 is

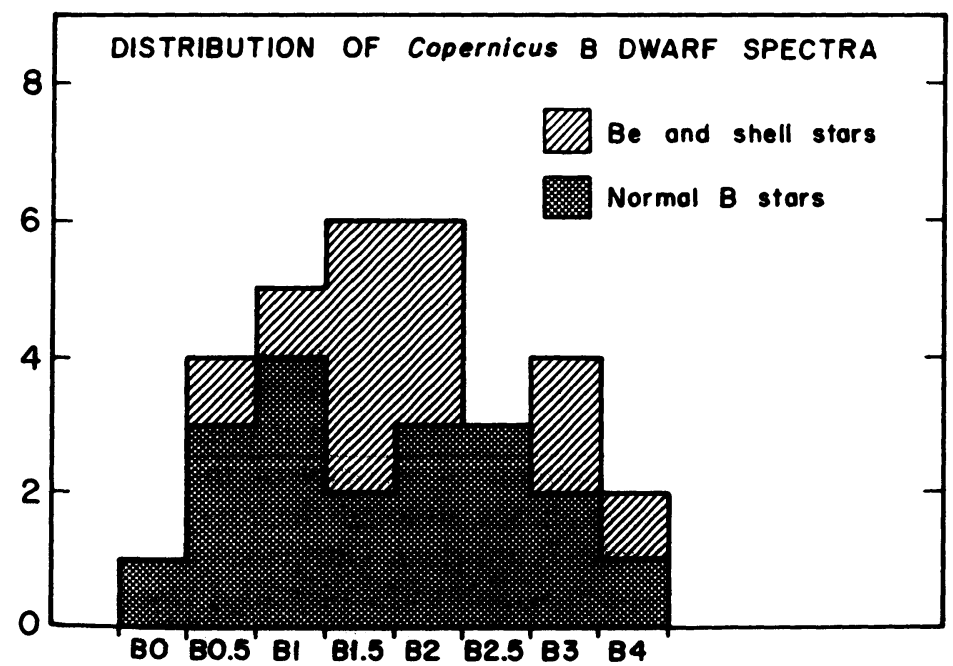

Fig. 1. The distribution by spectral type of the B-type dwarfs and Be stars considered in this survey. Included are only objects for which complete (or nearly so) U2 spectra are available, and for which the signal-to-noise ratio is reasonably high as far longward as the Si IV doublet at $\lambda 1400 \AA$. 
represented by at least one star. Many more objects in this category have been scanned with Copernicus, but only partial spectra were obtained, and these stars are not included here because data are available at most for only a few of the features commonly showing mass-loss effects. Also omitted from consideration were stars for which the quality of the data was too poor to allow a conclusion to be reached regarding the presence or absence of line asymmetries. This criterion primarily applied to the Si IV $\lambda 1400 \AA$ doublet, since this is in a wavelength region where the sensitivity of Copernicus is relatively low.

All the spectra were plotted at a scale of $4 \AA$ in. $^{-1}$, and careful comparisons were made in order to determine whether or not any indications of mass ejection were present. The wavelength regions studied included most lines considered in the survey of mass-loss effects in hotter stars (Snow and Morton, 1976); specifically, C II $(\lambda 1334.532 \AA), C_{\text {III }}(\lambda 1176 \AA), N_{\text {II }}(\lambda 1085 \AA), N_{v}(\lambda \lambda 1242.804,1238.821 \AA)$, O VI $(\lambda \lambda 1031.945,1037.627 \AA)$, Si III $(\lambda 1206.510 \AA)$, Si IV $(\lambda \lambda 1393.755$, $1402.769 \AA)$, and S IV ( $\lambda \lambda 1062.672 ; 1073 \AA)$.

Lines were considered to show mass-loss effects only if asymmetries were present which could not be attributed to blends with other nearby lines, or if large shifts were seen. Checking for blends was accomplished primarily by making comparisons among stars of similar spectral type. In the case of doublets such as Si IV $\lambda 1400 \AA$, this procedure was simplified by the requirement that any asymmetry due to mass-loss must be present in both lines, whereas blends would not be expected to affect both in the same way.

In Table I are listed the Be and shell stars included in this survey, along with relevant spectroscopic and photometric data. The spectral classifications are primarily taken from Lesh $(1968,1972)$ or Morgan and Keenan (1.973); the photometric data from Lesh $(1968,1972)$, Johnson et al. (1966), or the catalogue of Blanco et al. (1968); and the projected rotational velocities from Uesugi and Fukuda (1970) or Boyarchuk and Kopylov (1964). Although systematic inaccuracies may be present in the $v \sin i$ data, they should not affect the following discussion of the correlation of

TABLE I

Be and shell stars surveyed

\begin{tabular}{|c|c|c|c|c|c|c|c|}
\hline \multirow[t]{2}{*}{ Star } & \multirow[t]{2}{*}{ HD } & \multirow{2}{*}{$\begin{array}{l}\text { Spectral } \\
\text { class }\end{array}$} & \multirow[t]{2}{*}{$\boldsymbol{V}$} & \multirow[t]{2}{*}{$(B-V)$} & \multirow{2}{*}{$\begin{array}{l}v \sin i \\
\left(\mathrm{~km} \mathrm{~s}^{-1}\right)\end{array}$} & \multicolumn{2}{|c|}{ Observation date } \\
\hline & & & & & & Year & Day \\
\hline Cas & 5394 & B0.5 IVel & 2.58 & -0.20 & 300 & 1974 & $317-318$ \\
\hline$\alpha$ Eri & 10144 & B3 IV ev & 0.48 & -0.16 & 411 & 1972 & $280-285$ \\
\hline$\phi$ Per & 10516 & B1pe (III, V) & 4.06 & -0.04 & 450 & 1973 & $242-272$ \\
\hline \multirow[t]{2}{*}{$48 \mathrm{Per}$} & 25940 & $\mathrm{~B} 3 \mathrm{Ve}$ & 4.01 & -0.03 & 217 & 1974 & $352-354$ \\
\hline & 28497 & $\mathrm{~B} 1.5 \mathrm{Ve}$ & 5.60 & -0.23 & 340 & 1973 & $280-285$ \\
\hline$\zeta$ Tau & 37202 & B4 IIIp & 2.95 & -0.19 & 310 & 1972 & $319-323$ \\
\hline$\mu$ Cen & 120324 & B2 IV-Ve & 3.2: & -0.14 & 191 & 1975 & $142-143$ \\
\hline$\eta$ Cen & 127972 & B1.5 Vn & 2.3: & -0.20 & 300 & 1975 & $60-63$ \\
\hline$\chi \mathrm{Oph}$ & 148184 & $\mathrm{~B} 1.5 \mathrm{Ve}$ & 4.43 & +0.28 & 123 & 1974 & $242-243$ \\
\hline 59 Cyg & 200120 & B1.5 Ve2nn & 4.79 & -0.07 & 450 & 1972 & $289-293$ \\
\hline$v$ Cyg & 202904 & B2 $\mathrm{Ve}^{+}$ & 4.28 & -0.08 & 261 & 1974 & $306-308$ \\
\hline $31 \mathrm{Peg}$ & 212076 & B2 IV-Ve & 5.04 & -0.16 & 134 & 1974 & $295-296$ \\
\hline
\end{tabular}


mass-loss effects with projected rotational velocity. Also included is the date of observation of each star.

\section{Results}

Effects of mass ejection at speeds of several hundred $\mathrm{km} \mathrm{s}^{-1}$ were seen in a few stars in the survey. Of the normal B-type stars, only $\tau$ Sco (already reported by Rogerson and Lamers 1975) and $\theta$ Car (Snow and Morton, 1976) showed these effects. In both cases strong asymmetries were seen in $\operatorname{Si} I V \lambda 1400 \AA$ and $N$ V $\lambda 1240 \AA$, and in the case of $\tau$ Sco, also OVI $\lambda \lambda 1031,1037 \AA$ were broadened and asymmetric. The latter is of particular interest because, as pointed out by Rogerson and Lamers, $\mathrm{O}$ VI requires for its formation temperatures higher than normally expected in a B0 star photosphere; hence its presence in the spectrum may indicate that heating is taking place above the photosphere. Furthermore, it is suggested that the presence of highly-ionized species such as $\mathrm{O}$ vi helps to drive the high-velocity mass loss which is observed, via photon absorption in resonance lines. The general O-B star mass-loss survey of Snow and Morton (1976) provides some evidence that the high-velocity ejection of material may be linked to the presence of highly-ionized species.

Among the Be and shell stars, $59 \mathrm{Cyg}, \boldsymbol{\eta}$ Cen, and $\phi$ Per show marked evidence of mass loss. Si IV $\lambda 1400 \AA$ is strongly asymmetric in both $59 \mathrm{Cyg}$ and $\phi$ Per, and somewhat asymmetric in $\eta$ Cen. In addition, both 59 Cyg and $\phi$ Per show strong NV absorption, with extended short wavelength wings. Since most B1.5 stars show no $\mathrm{NV}$ absorption at all in $\mathrm{U} 2$ spectra, the fact that it is strongly present in these two objects is indicative that some unusual ionization mechanism must be acting. In the spectrum of $59 \mathrm{Cyg}$, the Si III $\lambda 1206 \AA$ line is also seen to be very asymmetric, further indication of mass flow. $\phi$ Per is discussed in much more detail by Plavec (this Symposium), as is $\eta$ Cen by Burton and Evans (also this Symposium).

Possible evidence for high-velocity mass loss is seen in the spectra of $\gamma$ Cas and HD 28497, in the form of Si IV asymmetries in the former case, and large shifts $(\sim 1 \AA)$ of the $\mathrm{Si}$ IV lines in the latter. As noted earlier, small displacements corresponding to expansion velocities of about $100 \mathrm{~km} \mathrm{~s}^{-1}$ or less are not being considered in this survey, but the apparent shifts in the case of HD 28497 are significantly larger, representing an outflow velocity of slightly more than $200 \mathrm{~km} \mathrm{~s}^{-1}$.

The fact that Bohlin (1970) has observed the C IV doublet in $\gamma$ Cas to show a P Cygni profile tends to support the suggestion here that mass loss is occurring in this star. Since $\mathrm{C}_{\mathrm{IV}}$ requires more energy for its formation than does $\mathrm{Si}$ IV, the two species may exist in different levels in (or above) the stellar atmosphere, so it may be that the rather different character of the $\mathrm{C}$ IV and $\mathrm{Si}$ IV profiles is due to a stratification of the velocity field. It is also quite possible that $\gamma$ Cas was more active at the time of Bohlin's (1970) observations than it was some five years later, when the Copernicus scan was made.

Si IV $\lambda 1400 \AA$ profiles of $\gamma$ Cas, 59 Cyg, HD 28497, $\eta$ Cen, and $\alpha$ Eri are shown in Figure 2. It is interesting to note that asymmetries may be marginally present in $\alpha$ Eri, a B3 star. Quite probably the $\lambda 1393 \AA$ line in this star is affected by blends, 


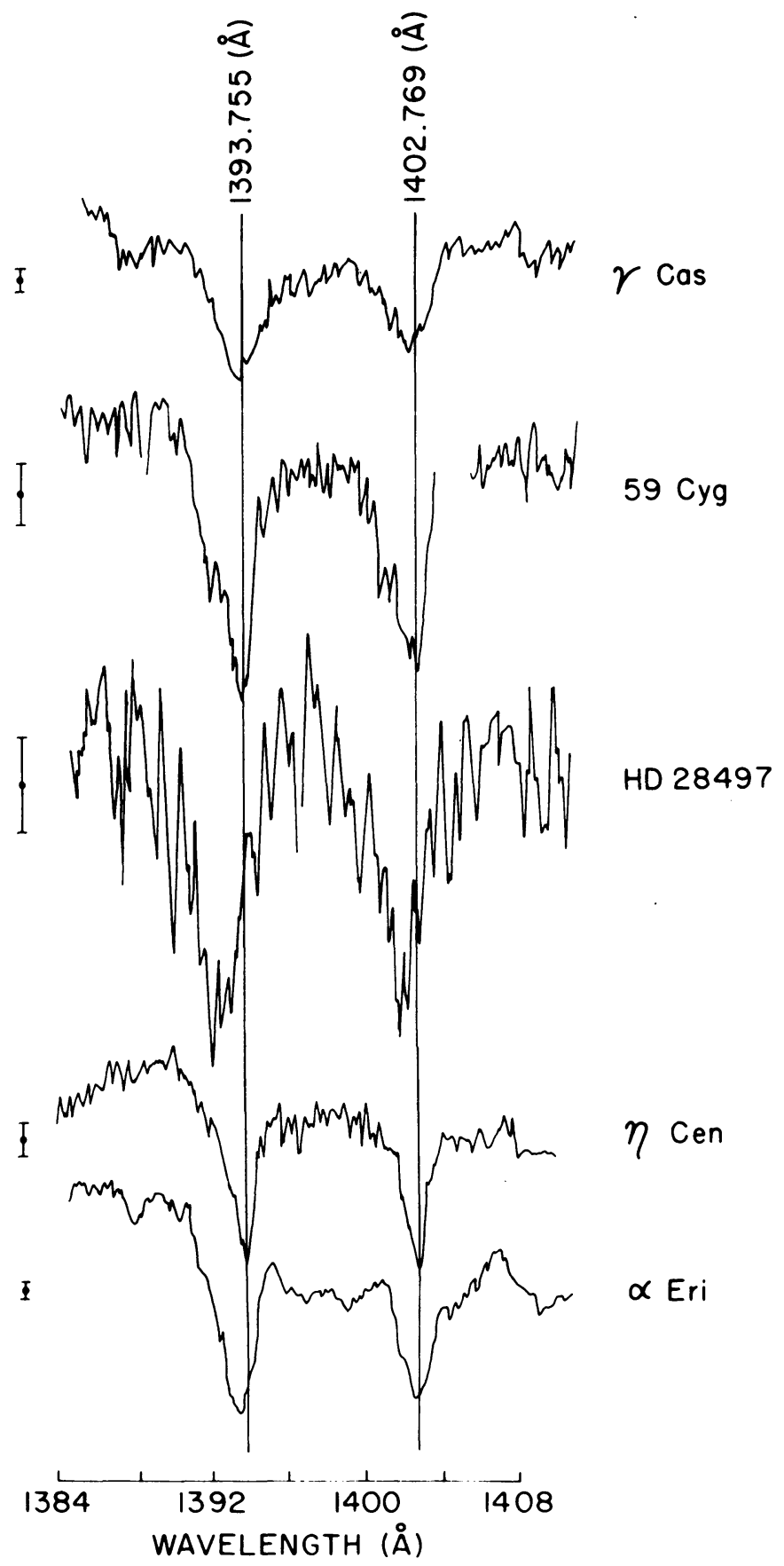

Fig. 2. Si IV $\lambda 1400 \AA$ doublet profiles in five of the Be or shell stars surveyed. The rest positions of the two lines are indicated. The error bars at the left represent the $2 \sigma$ rms noise at the continuum level due to photon statistics. Strong asymmetries are seen in $59 \mathrm{Cyg}$ and $\eta \mathrm{Cen}$, and a slight asymmetry appears to be present in $\gamma$ Cas. 
since it is more asymmetric than the $\lambda 1402 \AA$ line. However, the longer wavelength line itself may be asymmetric; if it is, its short wavelength edge represents a velocity of outflow of about $400 \mathrm{~km} \mathrm{~s}^{-1}$. It would be of interest to study these profiles with higher resolution to clarify the possibility that asymmetries may be present, because if so, this is certainly the latest dwarf star known to show evidence of high-velocity mass loss.*

None of the other stars in the survey showed effects of mass ejection. It is noteworthy, however, that a few objects were found which show apparent shell absorption features, but which are not classified as Be or shell stars on the basis of visual spectra.

\section{Discussion}

\subsection{MASS LOSS RATES}

Since normal dwarfs of spectral type later than B1 are not seen to have strong enough stellar winds to produce mass loss, while a few Be stars of type later than B1 do show its effects, it seems very likely that the Be phenomenon is related to the existence of mass ejection. As noted in Section 1, this is not a new idea, but evidence in the form of directly-observed high-velocity stellar winds has heretofore not been available. Copernicus data are consistent with the idea that $\mathrm{Be}$ star shells are formed from material ejected from the stars themselves.

The fact that only resonance lines show asymmetries in the Be stars having detectable mass flow is indicative that the mass-loss rates are not high, because in cases of high-volume mass loss, often some features arising from excited lower states (such as $C_{\text {III }} \lambda 1175 \AA$ ) show asymmetries or P Cygni profiles. The lack of a large population of these excited levels in the outflowing material probably implies that the density in this region is relatively low.

Furthermore, the comparative weakness of the mass-loss profiles is another indication that the rates are low. The stars having rates of mass ejection of order $10^{-6} M_{\odot} \mathrm{yr}^{-1}$ (e.g. Morton, 1967) show strong P Cygni profiles, while those of lower mass-loss volume display little or no emission, but only asymmetric absorption features (Lamers, 1976). Rogerson and Lamers (1975) find that $\tau$ Sco is losing material at a rate of $4 \times 10^{-9} \mathrm{M}_{\odot} \mathrm{yr}^{-1}$, while the present authors find for the $\mathrm{Be}$ star 59 Cyg a crude mass ejection rate of $10^{-10}-10^{-9} M_{\odot} \mathrm{yr}^{-1}$ (Snow and Marlborough, 1976). Certainly the other Be and shell stars showing line asymmetries do not exceed this rate, since their mass-loss profiles are no stronger than those of 59 Cyg.

Since $\tau$ Sco $(\mathrm{B} 0 \mathrm{~V})$ and $\theta$ Car (B0.5 Vp) both show mass loss but no Be phenomena, it may be that stars hotter than $\mathrm{B} 1$ which have radiatively-driven stellar winds are ejecting matter at such a high rate that shells are not able to remain in place, as suggested by Massa (1975) on the basis of visual data. The Oe stars, which can display very strong mass-loss effects, are difficult to understand in this context, however, since the presence of nearly stationary emission lines indicates that

\footnotetext{
* Note added in proof. New high-resolution (U1) scans of $\alpha$ Eri show that the Si IV $\lambda 1400$ lines have extended short-wavelength wings, indicating high-velocity mass flow in this star and further confirming the correlation shown in Figure 3.
} 
cirumstellar material must survive the rapidly streaming gas. Perhaps in these cases the visual emission occurs in a relatively dense region just above the photosphere, while the strong acceleration producing the high-velocity mass flow becomes effective at greater distances from the star.

Although the Copernicus data are consistent with the possibility that Be star shells are formed by radiatively-driven mass loss from single stars, it is also possible that the observed gas motions result from some other mechanism. One suggestion is that all Be stars are binaries with late-type secondary components, and that the shells form as a result of mass exchange (see Kriz and Harmanec, 1975, and references cited therein). It is known from Copernicus data that $\mathbf{P}$ Cygni profiles in cases of mass-exchange binaries can be very similar to those seen in single stars (e.g. McClusky et al., 1975).

\subsection{EFFECTS OF ROTATION}

It was suggested long ago (Struve, 1931) that the rapid rotation rates often observed in Be stars may play a role in the development of their extended atmospheres. The early concept of rotational break-up has in recent years been replaced by the development of models for radiatively-driven stellar winds (Lucy and Solomon, 1970; Cassinelli and Castor, 1973; Marlborough and Zamir, 1975; Rogerson and Lamers, 1975; Lamers, 1976). Since the existence of a wind in these models depends on the dominance of radiation pressure over gravitational forces for at least some ions or atoms, it is clear that a reduction in the effective gravity by rotation can enhance the formation of strong winds. Hence it might be expected that the presence or absence of high-velocity mass flow effects in Be stars is a function of their rotational velocity. That this is apparently so has already been pointed out in an earlier discussion of Copernicus data (Snow and Marlborough, 1976).

In Figure 3 is shown the distribution in the spectral class- $v$ sin $i$ plane of all the stars considered in the present survey, with filled-in circles or triangles to indicate respectively normal $\mathrm{B}$ dwarfs or $\mathrm{Be}$ stars not showing mass-loss effects, and open circles or triangles representing those which do. It is worth reiterating that evidence of atmospheric or shell expansion at velocities of about $100 \mathrm{~km} \mathrm{~s}^{-1}$ or less is not considered to represent mass-loss effects in the present context.

It is seen in Figure 3 that all the stars showing evidence for high-velocity mass loss lie in the upper left-hand portion of the diagram, consistent with the expectation that such effects should depend on rotational velocity and, of course, effective temperature. This more complete survey is consistent with the earlier suggestion (based on fewer data) that dwarfs in the spectral class range B1 to B3 or so lack sufficient radiative flux to drive strong stellar winds unless rotation sufficiently reduces the effective gravity (Snow and Marlborough, 1976). Hence Be stars may fall into a transition region where the degree of rotation determines the presence or absence of an extended atmosphere. It would be of very great interest to obtain high-resolution data on many of the Be and normal B stars which do not show mass loss effects in the U2 data, to determine whether milder stellar winds can be detected in stars below the apparent cut-off in Figure 3. It is possible, of course, that the lack of strong mass-loss effects in Be stars of relatively low $v \sin i$ is due to a geometrical effect, if these 


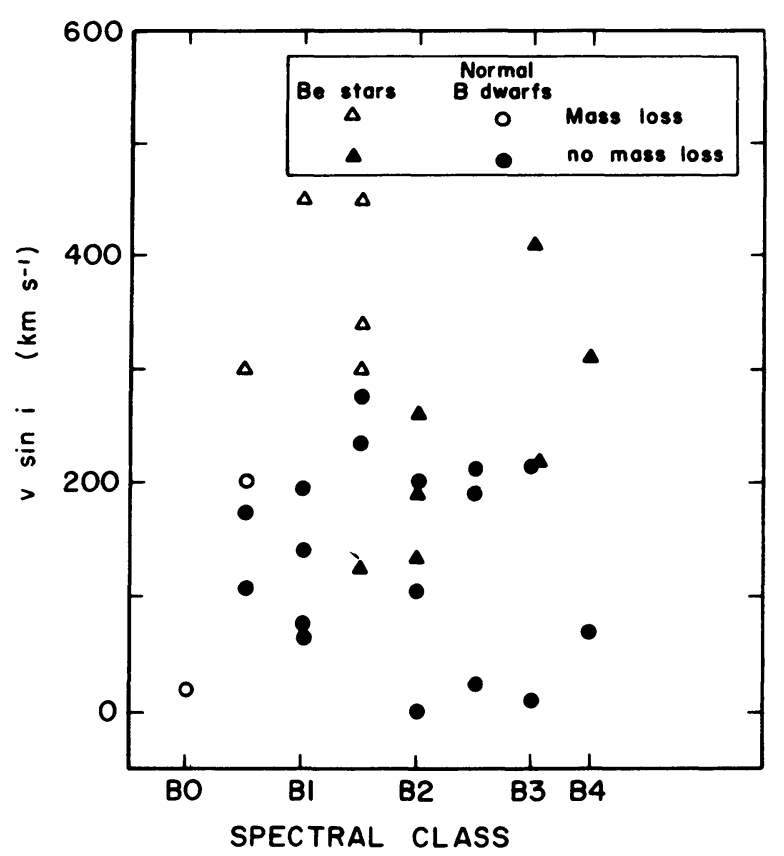

Fig. 3. Correlation of high-velocity mass loss with $v \sin i$ for all survey stars. The tendency of objects showing mass-loss profiles to lie in the upper left-hand corner is consistent with theories of radiatively driven stellar winds, as discussed in the text. All survey objects later than B0.5 which show high-velocity mass loss are Be or shell stars, supporting suggestions that rotation and mass loss play roles in producing the Be star characteristics.

objects are actually rapid rotators seen pole-on, because it may be that the outward mass flow is confined to the equatorial plane so that its effects on absorption lines can only be seen by observers in or near this plane. In any case, however, it is likely that the mass-loss rate is affected by the true rotational velocity, for reasons outlined above.

It will be interesting in future studies not only to see whether higher resolution data may reveal mass-loss effects in stars of lower effective temperature and $v \sin i$, but also to see whether many 'normal' early B stars with moderately high values of $v$ sin $i$ show shell absorption lines in the ultraviolet, as has already been noticed in a few cases.

\subsection{Correlation OF MASS-LOSS EFFECTS With PROPERTIES OF VISUAL SPECTRA}

It is of interest to consider whether or not any correlations exist between mass-loss effects and other properties of the visual spectrum. In particular is there any correlation of mass loss with Balmer emission line strength? In view of the fact that the emission lines in $\mathrm{Be}$ and shell stars are known to vary on a variety of time scales from hours to days and even disappear and reappear over periods of years, observations of the $\mathrm{H} \alpha$ line strength, or some other Balmer line, should have been obtained at the same time as the Copernicus data if meaningful correlations are to be 
found. Unfortunately no such results appear to be available for the stars in Table I. However, data on the $\mathrm{H} \alpha$ line strengths for some of the stars in Table I do exist corresponding to observations obtained by various workers over the period 1961 to 1975. In this section these observational results are examined to see if any trend exists between mass loss and $\mathrm{H} \alpha$ line strength.

Andrews (1968) observed many early-type stars during 1961 to 1963 using a spectrometer to isolate the region near $\mathrm{H} \alpha$. An estimate of the $\mathrm{H} \alpha$ line strength for those $\mathrm{Be}$ and shell stars in Table I in common with Andrews can be obtained by subtracting the observed $\mathrm{H} \alpha$ measure from the mean value for a star of the same spectral type and luminosity class obtained from Figure 5 of Andrews' paper. The estimates of the $\mathrm{H} \alpha$ line strength so obtained are given in column 2 of Table II and are such that the larger the numerical value the stronger is the $\mathrm{H} \alpha$ emission. The stars are arranged in order of decreasing strength of the $\mathrm{H} \alpha$ emission.

TABLE II

$\mathrm{H} \alpha$ line strength estimates

\begin{tabular}{llll}
\hline Star & $\begin{array}{l}\text { Andrews } \\
(1961-1963)\end{array}$ & $\begin{array}{l}\text { Feinstein } \\
(1970-1972)\end{array}$ & $\begin{array}{l}\text { Poeckert and } \\
\text { Marlborough } \\
(1974-1975)\end{array}$ \\
\hline$\chi$ Oph & 0.696 & 0.303 & 5.76 \\
$\phi$ Per & 0.688 & 0.249 & 4.50 \\
HD 28497 & - & 0.161 & 2.77 \\
$\mu$ Cen & - & 0.160 & - \\
$v$ Cyg & 0.627 & 0.135 & 3.67 \\
$\zeta$ Tau & 0.583 & - & 2.53 \\
48 Per & 0.497 & 0.156 & 2.94 \\
$\gamma$ Cas & 0.335 & 0.185 & 2.94 \\
59 Cyg & 0.329 & 0.092 & 1.66 \\
31 Peg & - & 0.064 & 2.00 \\
$\alpha$ Eri & 0.058 & - & - \\
$\eta$ Cen & - & 0.009 & - \\
\hline
\end{tabular}

Feinstein (1974) used interference filters to isolate hydrogen lines and measured line strengths photoelectrically for several hundred early-type stars. The observations were obtained during 1970 to 1972 . An estimate of the $\mathrm{H} \alpha$ line strength was obtained in a manner similar to that above by comparing the measured $\mathrm{H} \alpha$ index to that of a standard star of similar spectral type and luminosity class given in Table III of Feinstein's paper. Where more than one observation was available simple means were taken. The results are listed in column 3 of Table II. Stars observed by Feinstein but not by Andrews have been inserted in the appropriate place according to the $\mathrm{H} \alpha$ line strength, larger numbers again indicating stronger lines.

Finally estimates of the $\mathrm{H} \alpha$ line strength are available from the data of Poeckert and Marlborough (1976) and are listed in column 4 of Table II, larger numbers again indicating stronger lines. The data were obtained during 1974 and 1975. The $\mathrm{H} \alpha$ line strengths were derived from measures of linear polarization obtained with a polarimeter using narrow interference filters with a half power band-width of $5 \AA$. Because of the narrowness of the filter used the line strength estimates for stars with broad strong lines may be underestimated. 
It is clear from inspection of columns 2, 3 and 4 of Table II that the relative positions of some stars as regards line strength are not the same. Some of the rearrangement in column 4 relative to columns 2 and 3 may be due to underestimates of the $\mathrm{H} \alpha$ emission strength for cases where the emission is strong and $v \sin i$ large. Apart from this the rearrangement of some stars is probably an indication that the $\mathrm{H} \alpha$ emission strength varied over the period of observation. Indeed variations of the $\mathrm{H} \alpha$ index over the period 1970-1972 were reported by Feinstein for some of the stars he observed more than once. However for the majority of cases in Table II, large changes such as complete disappearance of emission lines do not seem to have occurred. Consequently some appropriate average will be taken as representative of the $\mathrm{H} \alpha$ emission strength at the time of the Copernicus observations.

The stars which showed marked evidence of mass loss from Copernicus data were 59 Cyg, $\eta$ Cen and $\phi$ Per and possibly $\gamma$ Cas and HD 28497. In Table II, these stars cover the range of strong to weak $\mathrm{H} \alpha$ emission but there does not seem to be any particular trend of the $\mathrm{H} \alpha$ strength compared to the degree of line asymmetry of $\mathrm{Si}$ IV $\lambda 1400 \AA$. Specifically 59 Cyg has one of the weakest $\mathrm{H} \alpha$ emission lines while $\phi$ Per shows one of the strongest. However both stars possess markedly asymmetric Si IV lines while $\gamma$ Cas, with lines which are not as asymmetric, has a $\mathrm{H} \alpha$ line of intermediate strength. Present data, therefore, do not appear to support any correlation of $\mathrm{H} \alpha$ line strength with mass-loss effects.

\section{Acknowledgements}

The portion of this work which has been carried on at Princeton has been supported by U.S. National Aeronautics and Space Administration contract NAS5-1810. The research at the University of Western Ontario has been supported by the National Research Council of Canada.

\section{References}

Andrews, P. J.: 1968, Mem. Roy. Astron. Soc. 72, 35.

Blanco, V. M., Demers, S., Douglas,.G. G., and FitzGerald, M. P.: 1968, Publ. U.S. Naval Obs. 21.

Bohlin, R. C.: 1970, Astrophys. J. 162, 571.

Bohlin, R. C.: 1975, Astrophys. J. $200,402$.

Boyarchuk, A. A. and Koplyov, I. M.: 1964, Publ. Crimean Astrophys. Obs. 31, 44.

Cassinelli, J. P. and Castor, J. I.: 1973, Astrophys. J. 179, 189.

Feinstein, A.: 1974, Monthly Notices Roy. Astron. Soc. 169, 171.

Hack, M. and Struve, O.: 1970, Stellar Spectroscopy of Peculiar Stars, Osservatorio Astronomico di Trieste, Trieste.

Heap, S. R.: 1975, Phil. Trans. Roy. Soc. London 279, 371.

Johnson, H. L., Mitchell, R. I., Iriarte, B., Wisniewski, W. Z.: 1966, Comm. Lunar Planetary Lab. 4, 99.

Kriz, S. and Harmanec, P.: 1975, Bull. Astron. Inst. Czech. $26,65$.

Lamers, H. J. G. L. M.: 1976, in preparation.

Lesh, J. R.: 1968, Astrophys. J. Suppl. 17, 371.

Lesh, J. R.: 1972, Astron. Astrophys. Suppl. 5, 129.

Lucy, L. B. and Solomon, P. M.: 1970, Astrophys. J. 159, 879.

Marlborough, J. M. and Zamir, M.: 1975, Astrophys. J. 195, 145. 
Massa, D.: 1975, Publ. Astron. Soc. Pacific 87, 777.

McLaughlin, D. B.: 1961, J. Roy. Astron. Soc. Can. 55, 13 and 73.

McCluskey, G. E., Kondo, Y., and Morton, D. C.: 1975, Astrophys. J. 201, 607.

Morgan, W. W. and Keenan, P. C.: 1973, Ann. Rev. Astron. Astrophys. 11, 29.

Morton, D. C.: 1967, Astrophys. J. 150, 535.

Plavec, M.: 1975, in preparation.

Poeckert, R. and Marlborough, J. M.: 1976, Astrophys. J., in press.

Rogerson, J. B. and Lamers, H. J. G. L. M.: 1975, Nature, 256, 190.

Rogerson, J. B., Spitzer, L., Drake, J. F., Dressler, K., Jenkins, E. B., Morton, D. C., and York, D. G.: 1973, Astrophys. J. Letters 181, L97.

Snow, T. P. and Marlborough, J. M.: 1976, Astrophys. J. Letters 203, L87.

Snow, T. P. and Morton, D. C.: 1976, in preparation.

Snow, T. P., York, D. G., Welty, D., and Hornack, P.: 1975, in preparation.

Struve, O.: 1931, Astrophys. J. 73, 94.

Uesugi, A. and Fukuda, I.: 1970, Contr. Inst. Astrophys. Kwasan Obs. Kyoto, No. 189.

Underhill, A. B.: 1966, The Early Type Stars, D. Reidel Publ. Co., Dordrecht-Holland, p. 226.

\section{DISCUSSION}

Heap: You say that you have found evidence for mass flow outwards at a speed near $1000 \mathrm{~km} \mathrm{~s}^{-1}$, and you found this by comparing the observed profile against some theoretical profile. What does the theoretical profile look like?

Snow: We did not assume any theoretical line profile. We simply assumed that the observed asymmetries are due to the presence of an optically thin, low-density stream of gas, so that no natural or collisional broadening takes place in the part of the absorption line which is formed in this stream. In that case the extreme short wavelength edge of the absorption profile represents material moving at the velocity given by the total displacement of that edge from rest.

Hutchings: Are your stars all high $v \sin i$ objects? That is, are they all seen equator-on?

Snow: All the Be stars which show mass loss have $v \sin i$ 's of $300 \mathrm{~km} \mathrm{~s}^{-1}$ or higher, and are all considered equator-on stars. However, our sample includes several B and Be stars of low and moderate " $\sin i$, as seen in Figure 3. The normal BO V star $\tau$ Sco has $v \sin i=20 \mathrm{~km} \mathrm{~s}^{-1}$, yet shows strong mass-loss effects. 